\title{
Optical twist measurement by scatterometry
}

\author{
A. Hertzsch, K. Kröger, M. Großmann \\ INNOVENT Technology Development, Prüssingstr. 27B, 07745 Jena, Germany \\ ah4@innovent-jena.de
}

\begin{abstract}
:
To ensure that seals do not leak one of the main requirements for the shaft surface is the absence of twist structures. Twist is a periodic, generally thread-shaped microstructure which is superimposed on the roughness of the shaft surface and takes the form of waviness in the axial and circumferential directions. Using an optimized scattering geometry light scattering leads to an intensified mapping of the periodic twist structure of the shaft surface. The optical filtering process reduces the speckle effect from surface roughness and produces a diffraction pattern from the twist structure. We use a rigorous diffraction model to calculate the diffraction efficiencies and to determine the twist parameters twist depth and twist period length. We introduce an optical scattering apparatus for fast and reliable measurements of the twist structure. A comparison between the experimental results of scatterometry and the experimental results of stylus measurements will be presented for a number of sealing shaft surfaces produced under different machining conditions. We discuss the limitations of the present model and indicate directions of future work.
\end{abstract}

Key words: Optical inspection, in-line, quality assurance, twist testing, scatterometry

\section{Introduction}

A safe and proven process for creating a functional surface for radial shaft seals is the plunge cut grinding. This process works without feed rate movement in axial direction and hence the texture of the grinding microstructure on the shaft surface should be perpendicular to the axial direction of the shaft. In this case no pumping process of oil is caused from the microstructure if the shaft rotates. But a change or fluctuation of the process parameters can easily produce an additional thread-like structure on the shaft surface: the twist. Inappropriate working conditions at the dressing process of the grinding disc or during the grinding process itself such as machine vibrations and bearing play can create such a twist structure on the shaft surface. So the main requirement of a ground surface is the absence of twist. However, the effects of the parameter changes or fluctuations of the grinding process are not exactly detectable and measurable. Therefore it is necessary to monitor the manufacturing process with a fast and reliable twist measurement method. Conventional twist measurement methods such as the thread method or stylus measurements are often too slow and in special cases not reliable enough. Optical diffraction analysis can visualize twist structures with amplitudes far below the amplitude of the surface roughness. For production-related optical twist testing on radial shaft seals we designed the Shaft Twist Tester STT $[1,2]$. The visualization of diffraction lines indicates the presence of twist structure. However, the user has to decide whether the picture shows diffraction lines or not. Now we want to introduce an automatic optical twist testing method. Images of light scattering distribution are recorded, filtered by image processing tools and evaluated for the automatic detection of diffraction lines. In the presence of diffraction lines the twist parameters period length and twist depth are calculated.

\section{The optical filtering process}

In the case of grazing illumination of the shaft surface in tangential direction the high coherence of the laser beam is used to amplify the display of periodic structures in the scattered light distribution of the reflected light. The periodic waviness in axial direction of the thread-like twist structure causes a conical diffraction pattern. Due to the curvature of the cylindrical shaft surface in tangential direction the conical diffraction pattern is widened to a stripe diffraction pattern (see fig. 1). The scattering of the roughness overlaps the diffraction pattern of the twist structure in a stochastic pattern (speckle pattern). By the grazing incidence of the laser light the speckle pattern is damped. Hence, the functionally 

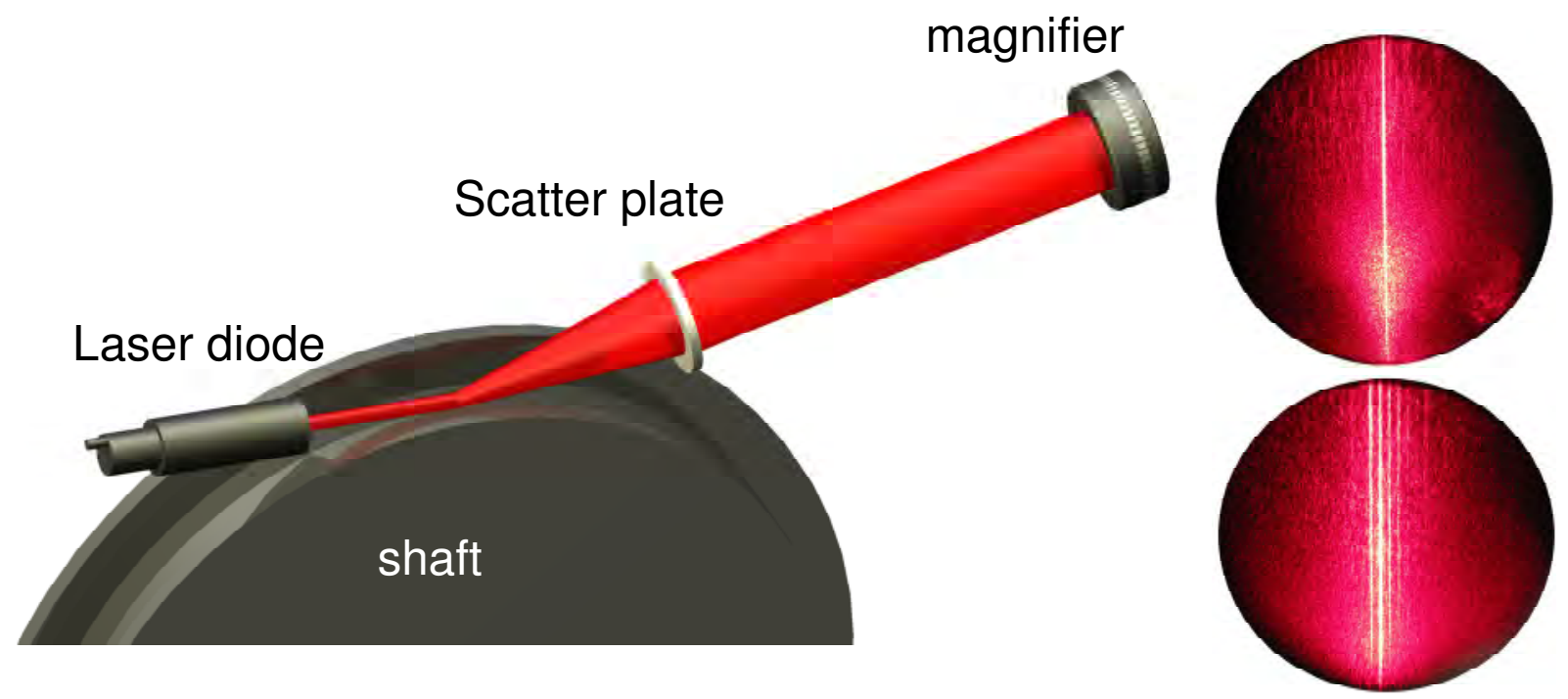

Fig. 1 : Scheme of the measurement system for the twist testing (left) and the test image of a twist free surface (at top right) and the test image of a surface with twist (at bottom right)

relevant overlap of roughness and waviness is visualized within the illuminated surface region. If the image of the light scattering shows one bright reflection line with a speckle pattern around the line (see fig. 1 at top right) the illuminated surface is without twist. If the image is characterized by some bright lines (diffraction stripes) a twist structure was produced at grinding process on the surface (see fig. 1 at bottom right).

The twist structures can be distinctly different over the whole seal seat circumference. The local dependency is characterized by varying portions of roughness and waviness. Consequently the periodicity of the twist structure is disturbed and the speckle pattern is the dominant part in the image of the reflected laser light. Furthermore, if the twist depth of the twist structures is small and/or the period length is large the diffraction lines can be discontinuous and blanketed from the stochastic speckle pattern. It is difficult in such cases to determine clearly whether diffraction stripes do exist in the image or if they don't. However, it is possible to stabilize this determination. If the shaft rotates during the optical twist testing the optical noise (speckle pattern) is minimized by superimposing the images. Depending on the local surface roughness the intensity and the location of the speckle varies at different positions of the shaft surface. The superposition of these different speckle pattern images takes the mean and reduces the contrast of the speckle pattern. On the other hand the disturbed diffraction stripes fall in the same region of the image and so the line shape and the contrast of the stripes are increased by superimposing the images. This
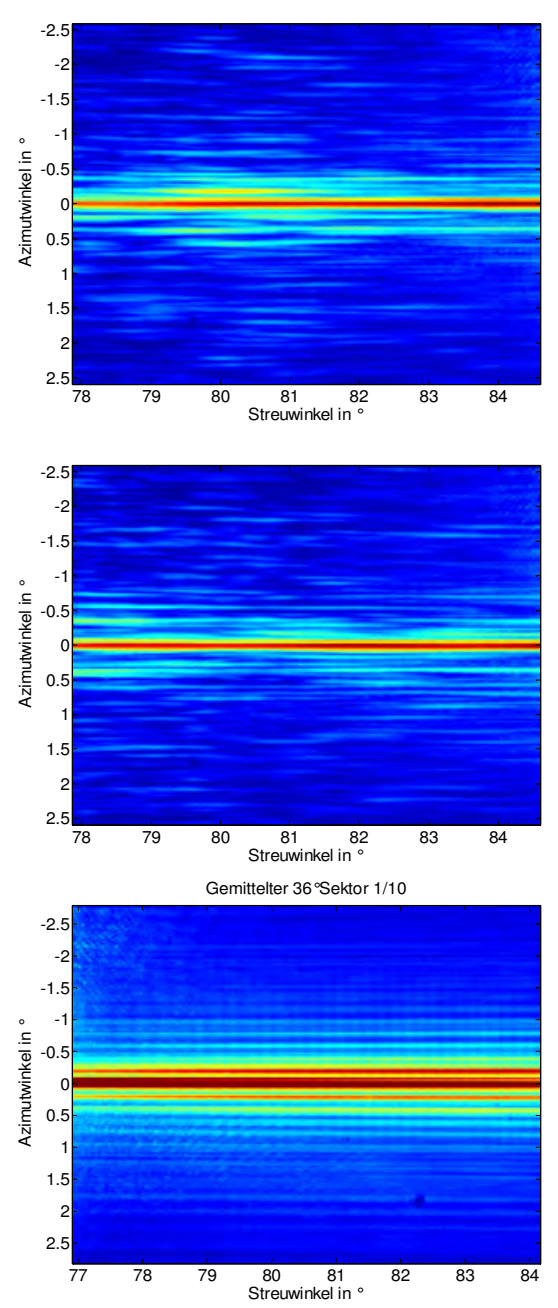

Fig. 2 : Scattering images of optical twist testing from ground shaft surface with small twist measured at arbitrary positions of the surface (top and middle) and measured and superimposed by rotating the shaft in an angular sector of $36^{\circ}$ (bottom) 
filter process is shown in figure 2. The ground shaft surface has following parameters: twist period length $\mathrm{DP}=200 \mu \mathrm{m}$, twist depth $\mathrm{Dt}=$ $0.26 \mu \mathrm{m}$, roughness parameters $\mathrm{Ra}=0.2 \mu \mathrm{m}$ and $R z=1.42 \mu \mathrm{m}$. The illuminated surface region is about $2 \mathrm{~mm} \times 2 \mathrm{~mm}$. On the top and on the middle of the figure 2 two scattering images of optical twist testing at arbitrary positions on this shaft surface illustrate the speckle behaviour. Diffraction lines are not
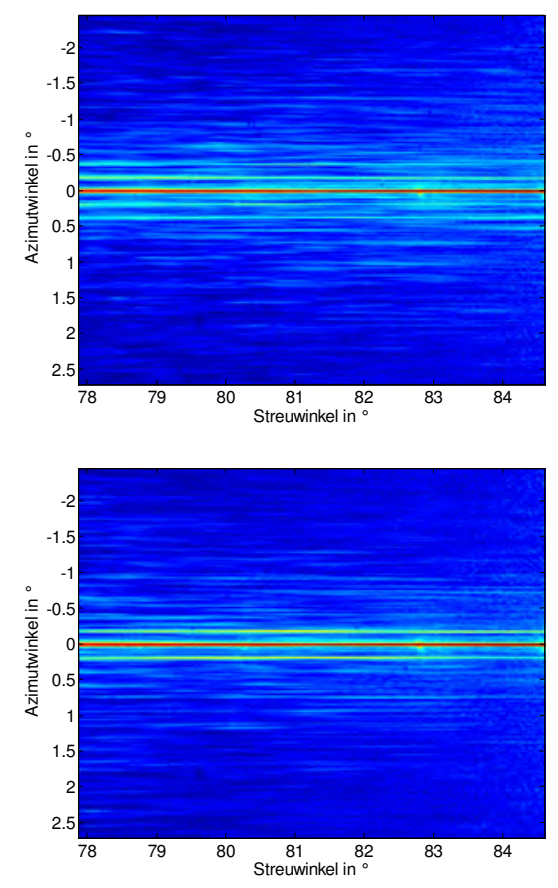

Gemittelter $36^{\circ}$ Sektor $1 / 10$

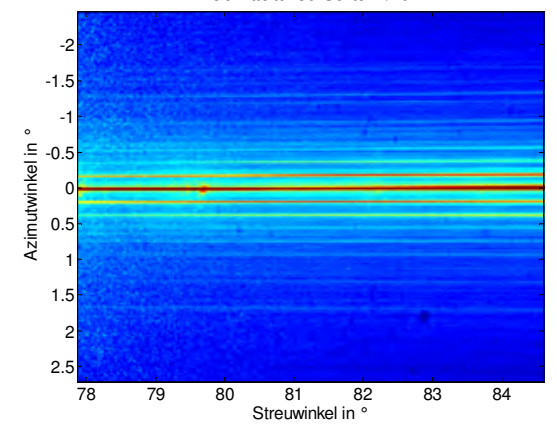

Fig. 3 Scattering images of optical twist testing from the ground shaft surface of fig. 2 measured with an illuminated region of $5 \mathrm{~mm} \times 5 \mathrm{~mm}$ at arbitrary positions of the surface (top and middle) and measured and superimposed by rotating the shaft in an angular sector of $36^{\circ}$ (bottom)

detectable. On bottom of figure 2 the superimposed image of 57 measured scattering images is shown generated by rotating the shaft in an angular sector of $36^{\circ}$. Clearly, diffraction stripes dominate in the image. The speckle pattern is minimized. Although the twist structure is small, the result obtained by superimposing uniquely identifies twist. To get global twist parameters of the closed shaft surface one revolution of the shaft during the twist testing is necessary. The best results are achieved if the shaft is held in a chuck or between centres and a motorized rotation stage provides the shaft rotation with high accuracy and stability. Recording the twist changes during rotation images of the light scattering pattern have to be captured at specific angular positions. By superimposing these images small twist structures are better detectable.

To increase the axial extent of the illuminated surface region a laser beam expander varies the diameter of the laser beam. So it is possible to intensify the diffraction pattern especially for twist structures with long period length. . More twist periods are illuminated and the contrast of the diffraction orders increases. The twist testing of the same shaft described above was realized with an illuminated surface region of about $5 \mathrm{~mm} \times 5 \mathrm{~mm}$. The results can be seen in figure 3 . The scattering images at arbitrary positions suggest that diffraction stripes are visible. However, in some images (for example on the top of fig. 3) the speckle pattern predominates. Once again the superposition of scattering images achieves the best result. The obtained image shows sharp and contrasty diffraction stripes.

\section{Calculation of twist parameters from scattering measurements}

A scattering model is applied to periodic surfaces in case the incident plane is parallel to the lay of the surface. Consider a scattering geometry shown in fig. 4. The laser beam illuminates the shaft surface in tangential direction at grazing incidence $\left(90^{\circ}-\Theta_{\mathrm{i}}\right)$. The main scattering occurs in the plane of incidence $x z$. If the shaft surface holds twist a diffraction pattern rises. Because the approximated onedimensional periodic structure is perpendicular to the incident plane the diffraction orders observed in the plane of screen at the distance $x$ lie on a circle (a circular cross section from the conical diffraction path). Each position of the diffraction orders is determined by the scattering angle $\Theta_{\mathrm{s}}$ and the azimuth angle $\varphi$. The locations of these orders are given by the following grating equations [3]:

$$
\begin{aligned}
& \sin \Theta_{s} \cos \varphi=\sin \Theta_{i}, \\
& \sin \Theta_{s} \sin \varphi=m \frac{\lambda}{D P},
\end{aligned}
$$

where $\Theta_{i}$ is the incident angle of the laser light beam, $\lambda$ is the wavelength of the laser and $\mathrm{m}$ is the diffraction order which is finite for a given periodic length DP. Substitution of equation (1) in equation (2) gives 


$$
D P=\frac{m \lambda}{\sin \Theta_{i} \tan \varphi}
$$

Equation (3) can be used to calculate the period length DP from the azimuth angle $\varphi$ at known angle $\Theta_{\mathrm{i}}$.

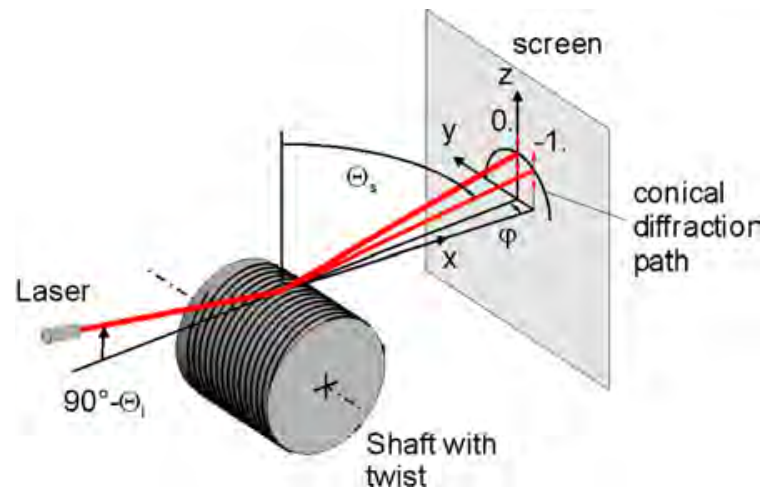

Fig. 4 Scattering geometry of optical twist measurement

As described above the curvature of the illuminated shaft surface causes the reflected light to diverge in z-direction and the spots of the diffraction orders to expand to diffraction stripes in z-direction centered at the conical diffraction path. The depth of the periodical structure is directly correlated to the intensity distribution of the diffraction orders. The deeper the twist structure is the more intensity falls into the higher diffraction orders. To determine the twist depth from this intensity distribution of the diffraction orders it is necessary to calculate the diffraction efficiencies of a grating model with the same period length. The grating depth of the model is varied in such a way that the simulated diffraction efficiencies fit in best with the measured intensity distribution of the diffraction orders. Because the incident angle of light is near grazing incidence near field contributions influence the diffraction. Hence we use the algorithm of rigorous coupled wave analysis (RCWA) to solve the completely vectorized system of the Maxwell equations. The grating profile is cut into slices of rectangular gratings for which the rigorous solution is known as a plane wave decomposition. The waves of every slice are coupled with each other in a matrix algorithm. To carry out this calculation we used the program UNIGIT [4]. A data base of diffraction efficiencies of gratings was simulated for a raster of period length DP and twist depth Dt to fit the measured diffraction intensities in a fast and easy way.

\section{Experimental results}

A number of shafts with typical twist structures produced by plunge cut grinding are tested. To compare the twist parameters with a reference method stylus measurements were realized for all surfaces based on the measurement instructions of the Mercedes Benz Norm [5]. The results of the twist parameters of these measurements are listed in table 1.

Optical twist measurements were made with the optical scattering apparatus shown in figure 5 . The sample is held in a lathe chuck which is turned in 572 angle steps per revolution with a motorized rotation. A laser beam is incident at an angle of $81^{\circ}$ on the shaft surface in tangential direction to the shaft. The illuminated region on the surface is approximately $5 \mathrm{~mm} x$ $5 \mathrm{~mm}$. A CCD-camera captures the scattering images. The scattering images are superimposed as described above in chapter 2 .

Tab. 1: Twist parameters of stylus measurements

\begin{tabular}{|l|l|l|l|l|l|}
\hline No. & $\begin{array}{l}\text { Diameter } \\
\text { in } \mathrm{mm}\end{array}$ & $\begin{array}{l}\text { Number } \\
\text { of threads }\end{array}$ & $\begin{array}{l}\text { Period length } \\
\text { DP in } \mu \mathrm{m}\end{array}$ & $\begin{array}{l}\text { Twist depth } \\
\text { in } \mu \mathrm{m}\end{array}$ & $\begin{array}{l}\text { Twist angle } \\
\text { in }\end{array}$ \\
\hline 1 & 15.0 & 144 & 280 & 0.123 & $40.57^{\circ}$ \\
\hline 2 & 34.9 & 54 & 66 & 0.641 & $1.86^{\circ}$ \\
\hline 3 & 19.1 & -8 & 200 & 0.266 & $-0.5^{\circ}$ \\
\hline 4 & 11.9 & 5 & 77 & 0.453 & $0.59^{\circ}$ \\
\hline
\end{tabular}




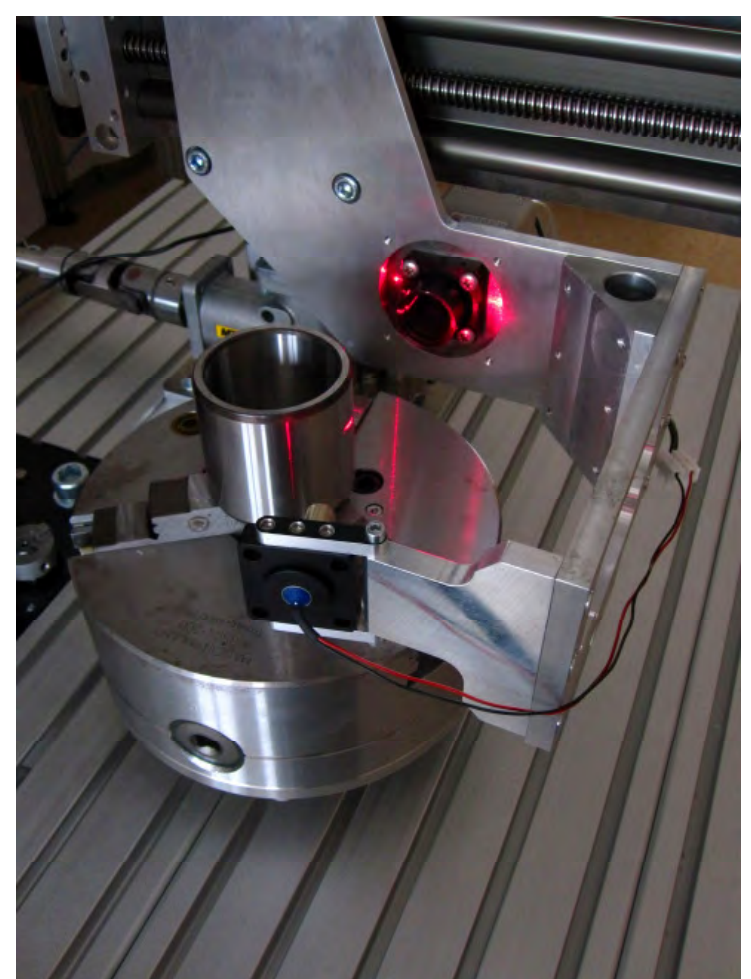

Fig. 5 :Optical scattering apparatus

Figure 6 gives an overview of the scattering results for all surfaces. The scattering images measured in an angle sector of $36^{\circ}$ were superimposed to typical diffraction patterns of the surfaces. Using the autocorrelation function for filtering the images and fitting the filtered diffraction patterns by a diffraction mask of the efficiency locations calculated from the grating equation (3) the period length of the twist structure DP is determined. The intensity distribution of the measured diffraction orders is used to find the simulated diffraction efficiencies of the RCWA-database (see chapter 3) with minimal deviation. Table 2 shows the intensities of the measured diffraction orders compared to the simulated ones and the twist parameters of the best fit.

The twist parameters determined by the optical twist testing agree well with the twist parameters made by stylus measurements. The best agreement is achieved for twist structures with large period length independent of the twist angle. Very small twist depth can be detected by this method. The diffraction efficiencies of twist structures with small period length are interfered by scattering and diffraction from double twist structures. These influences have to be analysed in future work.
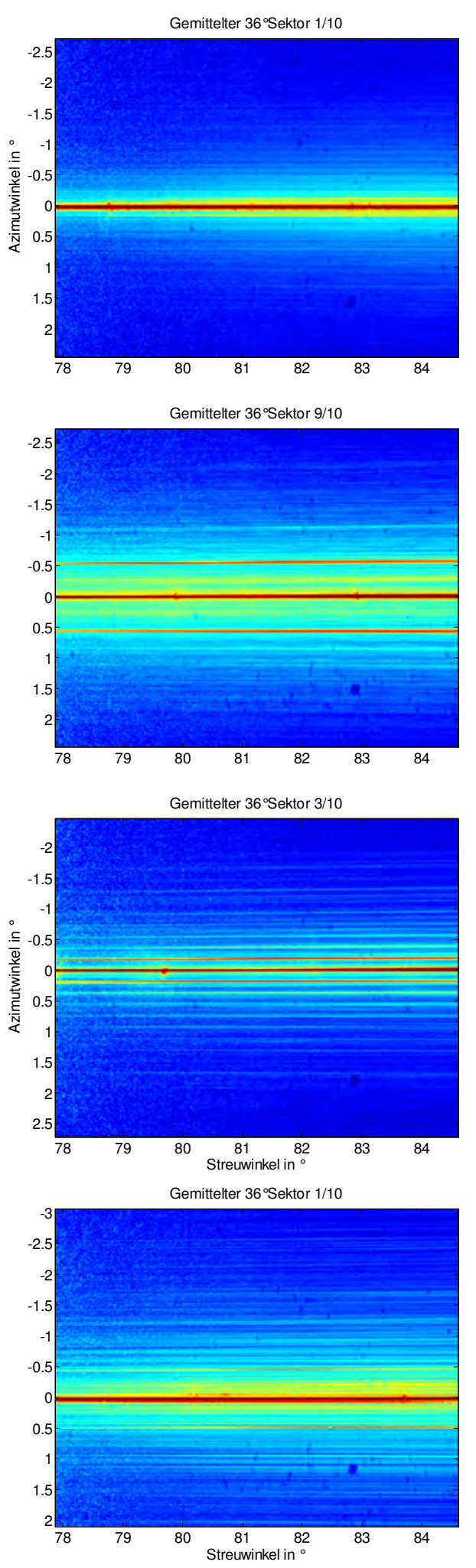

Fig. 6 : Scattering images of optical twist testing from surfaces No. 1 (top picture), No. 2 (second picture), No. 3 (third picture) No. 4 (bottom picture) of table 1 
Table 1 Diffraction efficiencies and twist parameters from optical twist measurement

\begin{tabular}{|c|c|c|c|c|c|c|}
\hline \multirow[t]{2}{*}{ No. } & \multirow[t]{2}{*}{ Analysis } & \multicolumn{3}{|c|}{ Diffraction efficiencies in $\%$} & \multirow[t]{2}{*}{$\mathrm{DP}$ in $\mu \mathrm{m}$} & \multirow[t]{2}{*}{ Dt in $\mu \mathrm{m}$} \\
\hline & & 0. & 1. & 2. & & \\
\hline \multirow[t]{2}{*}{1} & measured & 99.48 & 0.221 & - & \multirow[t]{2}{*}{280} & \multirow[t]{2}{*}{0.1} \\
\hline & simulated & 99.02 & 0.211 & - & & \\
\hline \multirow[t]{2}{*}{2} & measured & 88.71 & 5.44 & 0.34 & \multirow[t]{2}{*}{67} & \multirow[t]{2}{*}{0.4} \\
\hline & simulated & 87.34 & 5.7 & 0.36 & & \\
\hline \multirow[t]{2}{*}{3} & measured & 90.58 & 3.53 & 0.75 & \multirow[t]{2}{*}{200} & \multirow[t]{2}{*}{0.3} \\
\hline & simulated & 92.68 & 3.43 & 0.18 & & \\
\hline \multirow[t]{2}{*}{4} & measured & 90.47 & 2.76 & 0.89 & \multirow[t]{2}{*}{80} & \multirow[t]{2}{*}{0.27} \\
\hline & simulated & 93.63 & 2.98 & 0.15 & & \\
\hline
\end{tabular}

\section{Conclusion}

We introduce the optical twist measurement which can detect twist structures of shafts produced by plunge cut grinding automatically by analysis of the diffraction pattern. The twist structure can be distinctly different over the whole shaft circumference. The local dependency is characterized by varying portions of roughness and twist. By rotating the shaft und superimposing the measured scattering images the speckle effect is reduced and small twist structures are measurable by intensifying the diffraction pattern. The measured diffraction pattern can be fitted with simulated diffraction efficiencies of a grating calculated with the rigorous diffraction model RCWA. Hence it is possible to determine the twist parameters period length DP and twist depth Dt. The experimental results of typical twist structures show good agreement with stylus measurements.

\section{References}

[1] Hertzsch, A., Berghof, C., Großmann, M., Kröger, K.:,Fertigungsnahe optische Drallprüfung an zylindrischen Dichtflächen“, Kongress zur Getriebeproduktion (2011) Band II 283-292.

[2] Hertzsch, A., Großmann, M., Kröger, K.: „Optische Drallerkennung für die fertigungsnahe Qualitätskontrolle“, ATZ Produktion 3. Jahrgang, 05-06(2010) 40-44.

[3] Y. Fan: "Light scattering from periodic surfaces and turned surface roughness measurement", Ph.D. dissertation, University of Windsor, Windsor, 1992.

[4] UNIGIT: program for grating diffraction analysis, Ing.-Büro OPTIMOD, Ricarda-Huch-Weg 12, D07743 Jena.

[5] DIN EN ISO 25178-3 "Geometric product specifications (GPS) - Surface texture: Areal Part 3: Specification operators", (ISO/DIS 25178-3.2:2009); German version prEN ISO 25178-3:2009, (29.03.2010). 